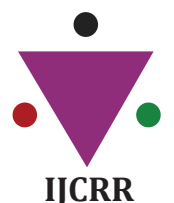

Section: Healthcare

Sci. Journal Impact

Factor: 6.1 (2018)

ICV: 90.90 (2018)

Scopus'

\title{
e-ICU's/Tele ICU's, it's Role, Advantages Over Manual ICU's and Shortcomings in the Current Perspective of Covid-19 Pandemic: A Critical Review
}

\author{
Naresh Rathod ${ }^{1}$, Aashishsingh Rajput ${ }^{1,2}$, Fouzia M $^{1,2}$, Jyothi D B ${ }^{3 *}$, Kalyani Patil ${ }^{4}$
}

'Resident, Department of Pharmacology, Vijayanagar Institute of Medical Sciences, Ballari, Karnataka, India; ${ }^{2}$ Resident, Department of Pharmacology, MGIMS, Sevagram, Maharashtra, India; ${ }^{3}$ Assistant Professor, Department of Pharmacology, Vijayanagar Institute of Medical Sciences, Ballari, Karnataka India; ${ }^{4}$ Epidemiologist, Tata Institute of Social Sciences, Mumbai.

\section{ABSTRACT}

The Coronavirus disease 2019 (Covid-19), is a viral pandemic that emerged in the Wuhan city of Hubei province in China, as a cross-transmission between bats and human beings. This is spreading exponentially across the globe, claiming lakhs of life worldwide and the counting is on. In view of this, there is increased risk and substantial burden on healthcare, and the Intensive healthcare community must prepare themselves to tackle this crisis. As this disease results in rapid deterioration of an individual's health status, without any warning signs, the burden on ICU's might be unexpectedly higher if more individuals become critically ill at the same time. In this context, the traditional mode of ICU operation might not meet the demands; hence technological advancements in the form of e ICU's or Tele ICU's may be the next visionary step. This model could tackle several interconnected e ICU's simultaneously with only a few dedicated teams of multidisciplinary specialist from a remote place and a very few bedside functionaries. This model may be advantageous to cut down the risk of infection due to ICU overcrowding, challenges with deficient PPE's, physical burnout and psychological breakdown of healthcare professional. This would also ensure round the clock uninterrupted services without compromising in the quality of care, viz a viz, substantially reducing the healthcare costs and financial burden on the management in the long run. Although met with technical challenges and shortcomings of lack of software personnel and round the clock high speed connectivity issues, the benefits of e ICU outweigh the risks involved. Hence the government, regulatory bodies and healthcare management must give a deep thought on revolutionizing age-old ICU models to technologically advanced e ICU's that may cater to a larger population with limited intellectual resources in a crisis like Covid-19 pandemic.

Key Words: e ICU, Tele ICU, Telemedicine, Covid-19, Pandemic

\section{INTRODUCTION}

In the current perspective, the world is facing a dreaded pandemic, with a major toll on the lives and economy across the globe. Coronavirus disease 2019 (COVID-19) is the third coronavirus infection in the past two decades, that occurred originally in Asia, after severe acute respiratory syndrome (SARS) and Middle East Respiratory Syndrome (MERS). COVID-19, emerged as an epidemic, to later spread globally as a pandemic in a very short period of time, it is presumed to have originated from the Wuhan city of china in the Hubei province, due to cross-transmission between bats and humans. History traced some major pandemic like the Spanish flu in 1918, which caused over 20 to 50 million deaths worldwide. In the year 1957-58 Asian flu pandemic occurred, which was triggered by influenza A (H2N2) virus's new strain, thought to have originated from East Asia, claiming the lives of over 1.1 million individuals worldwide. In 1968, Hong Kong flu pandemic triggered by new strain of $\mathrm{H} 3 \mathrm{~N} 2$ virus claimed lives of 1 Million people across the globe. 2009-10 the world witnessed swine flu pandemic (H1N1), which was triggered by a new strain of similar virus thought to cause Spanish flu. This pandemic affected almost 700 million to 1.4 billion people, but the mortality rates were comparatively lesser. Presently in May 2020, 5 million people have been reported to be infected with Covid-19, with death tolls touching a figure of 3 Lakhs and counting. ${ }^{1}$

Symptoms of Covid-19 disease vary from individual to individual depending upon the viral load and immunity profile, it

\section{Corresponding Author:}

Dr. Jyothi D B, Department of Pharmacology, Vijayanagar Institute of Medical Sciences, Ballari, Karnataka, India. Mobile: 8105134210; Email: drjyo6@gmail.com

ISSN: 2231-2196 (Print)

Received: 18.05 .2020
ISSN: $0975-5241$ (Online)

Revised: 12.06 .2020
Accepted: 02.07 .2020 
may range from fever, fatigue, tiredness and dry cough. The patient may also complain of malaise, nasal congestion, sore throat, headache, difficulty in swallowing, dysgeusia, diarrhea, anosmia, tinnitus, breathlessness and chest pain. Few individuals may get infected with this disease and still remain asymptomatic or with mild flu like symptoms, but they are potential candidates for transmission to other humans, it is presumed that almost $80 \%$ of the people recover from illness without needing specialized intervention, whereas the rest 20 percent, would invariable land up in severe complications like, severe hypoxemia, failing respiration, ARDS, followed by cytokine storm, leading to vascular hyper-permeability, multiorgan failure and eventually death of the individual. Elderly people with chest and cardiac co-morbidities like cardiac disease, high blood pressure, diabetes and immunecompromised states are more likely to succumb to the illness and mortality among these people are generally higher. These individuals require specialised care and prompt intervention in an ICU setup for an approximate period of 7 to 14 days or higher, depending upon the criticality of the patient. ${ }^{2}$

As Covid-19 is a highly contagious viral pandemic, with tolls rising exponentially combined with inherent challenges like global shortage of medical masks and respirators, Personal Protective Equipment's (PPE), deficient healthcare staffs, Shortage of ICU beds and increase in the workload, burn out, stress, medical leaves, quarantine of professionals post exposure to Covid-19 and the lack of technological advancements across ICU's pose a grave challenge in management of terminally ill patients requiring ICU care.

Tele-ICU/eICU models are already operational in countries like, Italy, Japan, Malaysia, USA for treating various emergencies condition even before the outbreak of Covid-19 pandemic. Although these countries were well-versed with the technical and procedural management of eICU still some countries like Italy, USA they were unable to accommodate the vast majority of critically ill patients of Covid-19, which demanded treatment and intervention in an ICU setup. This could be due to lack of extensive availability of eICU across the country or deficient skilled man power to operate them or both. In Indian healthcare system eICU concept is operational in few hospitals Fortis and Apollo hospitals located in metropolitan cities since a couple of a years ago. This concept has not gained momentum due to cost consideration, appreciation of technological advancements in management of critical care illness, lack of skilled professionals, and lack of need for implementation of this model on a larger scale in a revenue constraint environment. In a deadly pandemic like Covid-19 which is highly contagious and easily transmissible by airborne route and through fomites maintaining social distance and restricting undue doctor-patient interaction is considered the best practice. In such scenario the role of eICU is much appreciated which cuts across most of the risk factor in transmission of Covid-19. Hence, to manage pandemic effectively more number of eICU needs to be established across pan India. That would enable a limited number of skilled staff to manage critical care emergency in vast majority of Covid-19 infected individuals. But this may be faced with challenges of budget and infrastructural constraint in an economic downfall.

\section{PANDEMIC AND ITS TOLL ON HEALTHCARE WORKERS}

Throughout the globe, the healthcare workers fighting this situation on the frontlines have a grim story to tell about the scarcities of life-saving health supplies, including PPE's and stories of death. Across the globe, social media is showcasing the hazardous consequences upon the healthcare professional despite the usage of PPE's. Mortality of healthcare professionals are on the rise and counting. Europe and Asian statistics estimate that around 3000 health professionals in China, 6000 in Italy and 5400 in New York health workers are infected due to COVID-19. ${ }^{16}$ While America being in the same path still have the unclear statistical data of COVID-19 infected healthcare professionals because they lack the key data which clarifies the exact figures at the moment. Country like India with a population of 1.3 billion people tends to live in the different states with different socio-economic backgrounds, in spite of having quick disaster alleviation plan of announcing early lockdown, social distancing, quarantine and isolation plan, hygiene management, and instructions of wearing mask is still seen in steady elevation in the number of positive cases of COVID-19 which are inclusive of healthcare professionals. In spite using full or minimum Personal Protective Equipment's at their expense. This proves that the virus is highly contagious and easily transmissible from one individual to another or through objects/surfaces to individuals, not clearly described yet. More study and research need to be done in order to understand the precise mechanism of transmission of virus thoroughly. ${ }^{3}$

In the current scenario, despite the non-pharmaceutical interventions like social distancing and etc., minimizing the load of virus should be the key strategy to reduce the transmission. For this, there should be patient doctor interaction without compromising the safety of the already deficient healthcare professionals and its subsequent quality of care. This should overall help to reduce the viral load and transmission of infection. eICU plays an important role in this context, that can majorly tide across these inherent barriers.

\section{GLOBAL CHALLENGES WITH REGARDS COVID-19}

The world is facing a global shortage in the availability of medical masks and respirators for the warriors tackling the 
disease in the forefront and the general public as a whole, this threatens the efforts to prevent the transmission of the disease. N95 respirators that don't fit the facial contours might not provide necessary protection and may increase the risk of forefront warriors succumbing to the infection. There is a global short of PPEs, that further adds the risk of viral infection; on the other hand self-contamination during the removal of PPEs poses another challenge. In ICU set up, Viable virus on the surface of the mask, PPEs, mobile phones, or any other medical equipment increases the chances of nosocomial transmission using fomites. In manually operated ICUs, frequent visits by healthcare professionals and visitors, further increase the risk of infections, that may further transmit to the community. ${ }^{4,17}$

Airborne infection isolation rooms with essential negative pressure are not available universally, specially in a resource-constrained environment. The upsurge of critically ill Covid-19 patient might occur rapidly and may result in a state of shock for the management, with the deficient resource available. Low- and middle-income countries face challenges with the availability of ICU beds that are usually deficient. On the other hand, economically well-developed countries might also face challenges with the availability of ICU beds in a sudden outbreak of Covid-19. Any attempts to increase the availability of ICU for the treatment of critically ill patients also demand the availability of basic infrastructure like ventilators, consumables and drugs, which might fall short of supply in the pretext of the pandemic like Covid-19, and presently as well know the world is facing a short supply of ventilators. ${ }^{18}$

Just increasing the ICU beds might not serve the purpose; this may result in the availability of arms without an army to battle this pandemic, hence availability of skilled staff to manage an ICU is the primary concern. Unequipped ICUs, might result in increased mortality. There is a constant risk of loss of staff to medical illness, quarantine, unprotected exposure to Covid-19, that can affect the psyche and mental composure of the available staff, and during outbreaks like Covid-19, healthcare professionals are generally under mental stress and may land up in depression and anxiety. ICU triage protocols might get tampered if the rate of admission upsurges as the pandemic spreads. Hence in such a scenario, technological advancement in the form of telemedicine/ tele ICUs/e ICUs can help monitor a large number of ICU patients with limited staff and uninterrupted round the clock quality services that cuts through the inherent challenges across ICU setups. ${ }^{4}$

\section{TELEMEDICINE AND REMOTE SENSING TECH- NOLOGY IN PRETEXT OF COVID-19}

Telemedicine with addition of remote sensing technology will limit the amount of exposurea healthcare professional might get exposed during his/her routine Covid19 duties. Using this technology, we can manage unethical admission, proper bed utilisation, drugs and other resources.Looking at the contagiousness of the covid-19 infection it may well be apprehended that even if a slight proportion of population across the globe get infected it would result in thousands of people becoming critically ill which may require management admission in intensive care unit setup. Unfortunately, many countries in the world including developing and underdeveloped countries might not have adequate ICU bed, to tackle sudden upsurge of COVID-19 cases. In this regard the global ICU community must robustly prepare itself for the sudden spike of COVID-19 cases that may over burden and cripple the current functionality of ICU setup across the world. This would be possible if the conventional ICU across the globe would update itself with the modern technological advancement in the form of eICU or TeleICU incorporating artificial intelligence, embedded and remote sensing technology. Such a setup would let us optimize the workflow pattern in advance, enable us to rapidly diagnose and isolate the cases and subsequent clinical management using limited resources. The governing bodies across countries including the administrators and policy makers must join hands with ICU professionals to prepare an extensive and updated plan that would focus towards bed capacity across intensive care unit. ${ }^{5}$

\section{WHAT IS TELEMEDICINE?}

Telemedicine is a science that deals with the exchange of medical information from one site to another using advanced and sophisticated technologies that incorporate artificial intelligence, embedded and remote sensing devices for better healthcare delivery. ${ }^{21}$ The upsurge of Telemedicine in recent years has changed the clinical practice of all healthcare professionals and workforce which has resulted in changing of professional roles either because of new work flows or active monitoring strategies. eICU enables team members from varied discipline to work closely, it encourages communication among themselves about an individual patient and enables them to work as a team.

\section{CORE COMPONENTS OF AN EICU (TELE-ICU) MODEL}

\section{The first core component of an eICU essentially consists of a:}

- A multidisciplinary care team consisting of an intensivist, Physician, nurses, physician assistants which are well trained in acute and critical management.

- Also, critical care nurses, pharmacist, respiratory therapist, health administrator and an information technologist working congruently and comprehensively towards the management of an individual or a group of patients within the connected chain of eICU.? 


\section{Second essential core component of an eICU care revolves around the following values:}

- Round the clock availability of intensivist to the ICU patients across multiple connected hospitals through a remotely operated, intensivist led multidisciplinary team.

- eICU value patient rights with regards to safety, privacy and confidentiality.

- eICU encompasses the importance of family care, prevention of risk of exposure and diseases. It encourages collaborative team functioning with sound decision making backed by evidence-based clinical medicine for the betterment of healthcare facilities rendered to the patient.

- eICU standardizes the care of management with predesigned protocols that would serve as a basis for customized management of individual patients based on their disease condition and treatment demands.

- eICU enhance the performance of individuals in the multidisciplinary team by cutting of the distractors from their conventional methods of practice which inevitably causes hindrance to better healthcare delivery.

- eICU is a need-based concept that emerges from scientific enquires questioning the limitation of conventional clinical practice. ${ }^{4}$

\section{The third component of elCU encompasses:}

- Telecommunication gives an enormous opportunity to the healthcare team to reach out to patients from one location to another without geographic limitation. It is also available at the hospital ICU nurses station useful for visual communication with on-site doctors, nurses, and respiratory therapists, and family consultation if needed. ${ }^{8}$

- Teleconferencing uses audio and visual devices which can be used for consultation, enabling therapeutic management across the globe. Virtual rounds can be conducted 3 to 4 times per day. Providing medical expertise wherever needed. ${ }^{9}$

- Clinical Information system holds the database of patient medical history and radio scans, which can be accessed anywhere anytime.

- Computerised decision support is an evidence-based science-based on clinical based guidelines and standard protocol improve ICU patient care and improvising cost-benefit decision for minimizing variations in the treatment methods.

- Hospital information system improves clinical care \& patient safety, Quick \& coordinated care. Helps in reducing re-admissions, reducing waiting times and also giving cost-effective treatment.

- System databases and Improved Particle Swarm Optimisation (IPSO) that is applied to build a personal physiological signal sensing system based on sensing cloud architecture using cloud equipment technology. ${ }^{4}$

\section{CHALLENGES OF MANUAL ICUS IN MANAGEMENT OF COVID-19}

- In a manual ICU setup, critically ill patients are managed manually with the help of healthcare professionals, that need to be present physically for examination, diagnosis, treatment and subsequent interventions. Endotracheal intubations and other aerosol generating procedures need to be conducted in person, increasing the viral load, and chances of infection in an overcrowded ICU facility.

- Healthcare professionals managing a manual ICU essentially comprises of an intensivist, an emergency nurse for carrying out tracheal intubation and ventilation support, a physician, a physician assistant and sometimes a pulmonologist to look into the general condition of patient and decide further therapeutic management. A radiologist along with his technician for on-site performance of scan and x-rays, a nurse for drawing blood samples, for insertion and removal of catheters, stabilizing IV lines and for continuous monitoring of vital parameters. Paramedics, ICU technician for looking into logistic of ICU functioning, a pharmacist for dispensing medication, sweepers, helpers, cleaners and allied healthcare professionals to maintain hygiene and sanitation. These entire team needs to work on a shift on and shift off basis, hence at any given point of time, three sets of such dedicated and trained personnel needs to be available for ensuring uninterrupted, round the clock services. Thus a huge number of professionals are required for running one single ICU. Which the government or the healthcare bodies, cannot afford in a pandemic situation like Covid-19.

- This means a lot of manual movements in and out of the ICU setup would happen on a daily basis, which can serve as a potential threat for transmission of the viral infection from one site to another.

- Managing such a huge team of healthcare and allied professionals, incur huge costs with regards to the renumeration, training, providing for protective equipment's and their resting facilities, which would have a huge impact on the finances of the management in a resource deficient, lockdown scenario.

- In a manual ICU setup, repeated investigations, repeated consultations are a norm to gauge the health status of a patient. Thus, frequent interaction between the doctor and patient might render the physicians and associated personnel, to unwarranted exposure of increased viral load, thereby increasing their chances of succumbing to Covid 19.

- Training of these healthcare professionals, needs to be carried out regularly, with fresh staff recruiting to supplement the existing ones, in case of a medical emergency, medical leave, post-exposure quarantine and allied challenges of the existing ones. This adds up to the already existing financial burden. 
- Training sessions in a pandemic scenario are rapid, quick and accurate, that demands quick learning professionals across healthcare. All the medical personnel involved in a manual ICU needs to be trained and streamlined in the protocol and procedure which are pre-designed and standardized in a short period of time. This may increase the chances of grave error while executing their duties due to differences in understanding and retention capabilities of procedural aspects of COVID 19 management. But as these personnel are already under stressful conditions, individual variability of learning is inevitable. And the same concept might be perceived differently by different professionals undergoing training due to subjective variations. This would result in manual errors of monitoring and treatment, which can indirectly add up to the mortality of critically ill ICU patients.

- Work stress, burnout, and psychological issues of the working professionals, might hamper their decision making on the go, that might be deleterious for the ICU admitted patients, indirectly contributing to compromised quality of care and mortality. ${ }^{4}$

\section{ADVANTAGES OF E ICU'S OVER MANUAL ICU'S}

- In an eICU, the treating physician can manage the patient from a distance place, anywhere in the country, using a monitor which can interact with the patient directly, using telemedicine facility. The aerosol generating procedures and other interventions can be carried out with minimal staff under the supervision of other specialist, hence this helps us to reduce the overcrowding within the ICUs and reduces the risk of transmission of viral infection like Covid-19.

- In an e ICU setup, the dedicated team of professionals manage the patients from their respective place of residence or from a distant facility using remote sensing technologies, and it enables them to management more than one ICU, which are interconnected digitally with the help of a very few in house ICU staffs who are well trained in following the necessary protocols and commands. This helps to cut off the barriers of deficient PPEs, and other infrastructural challenges that accompany.$^{10}$

- e ICU's help us to cut down the unnecessary movement in and out of the ICU facility which would prevent unnecessary risks of transmission due to limited movements. It also prevents burnouts of the specialist and allied personnel. These can manage and monitor a large number of patients from their respective place of ease and comfort, enabling swift and intellectual functioning at their best potential in the absence of stress factor.

- eICU's enable Multidisciplinary approach of management, as it would enable the specialists and team to frequently interact with each other and make decisions for the best management of the patient in question. This would help to remove human errors and lessen the mortality rates.

- Although, setting an e ICU would incur, some additional charges during the phase of installation, with regards to the installation of the biosensors, monitors, remote sensor and telemedicine devices, but once connected with a large number of related ICUs may the setup to be managed by a few competent and technologically advanced set of ICU professionals, that would save on the overall finances in the long run, yet providing the best quality of care with minimum errors in patient management.

- The biosensor and other devices, give nearly accurate readings on a continuous basis and the personnel can look into the vitals whenever required, hence unnecessary movement of the treating nurses can also be reduced, protecting them from risk of transmission. And interventions are sorted only when required on an emergency basis as per the decision of the monitoring specialist.

- The team of dedicated staff for managing eICU 's can undergo training in the beginning and once well versed, they can carry out the functions without any difficulty. And then can train the incoming set of new personnel as necessity demands. Hence ongoing training costs and infrastructural demands could be taken care of quickly by this method of functioning.

- eICU 's eliminated the barrier of repeated in person consultation, because in this set up, the treatment physician or any other personnel can look at the vital parameters or speak to the patients as an when required, from their place of convenience. This would enable personalized quality care and will also maintain confidentiality, ethics and professional conduct.

- eICU indirectly removes a lot of psychological stress on the healthcare professionals, working in a highly risky environment, by enabling them to function from their location of comfort and intervene in person, only when required. This substantially eases the barriers among the doctor and their family lives, while delivery quality of care on a larger perspective. ${ }^{11}$

\section{HOW DOES EICU/TELEICU FUNCTION}

The functioning protocol of an e ICU is depicted in Fig.1, which shows the inherent procedures and algorithms involved. On one end is the designated ICU with beds and patients and necessary telemedicine devices involved, Every patient is tagged with an identification code that the networking clients would identify, which would then route using internet or router facility to the server devices like the mobile phones, monitors etc. these are accessible by the team of qualified professionals at any point of time, and at 
any place. Hence, using this protocol, a physician or an associated healthcare professional can directly interact with the designated patient using the monitor as an interface and carry out the necessary consultations. ${ }^{12}$

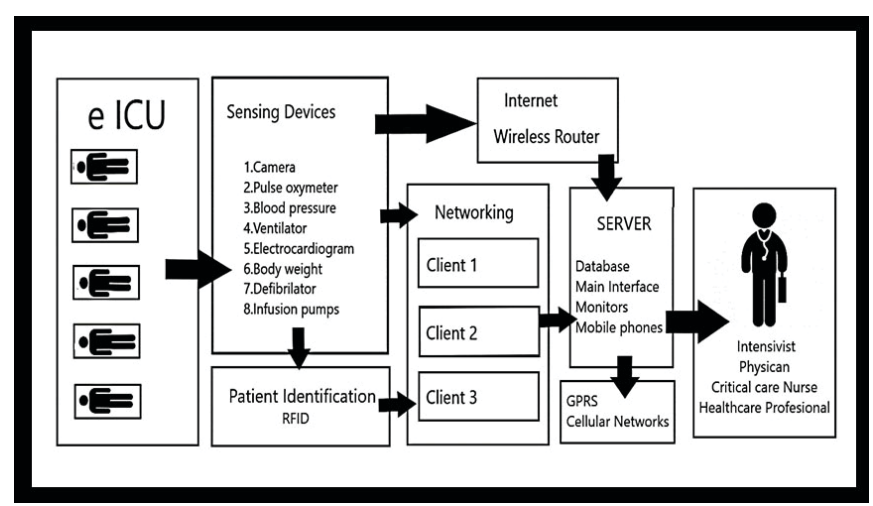

Figure 1: An algorithm to demonstrate the procedural protocols of the functioning of an e ICU.

\section{DISADVANTAGES OF EICU}

- Although installing an eICU setup proves beneficial in the long run but it could incur substantially higher cost for installation of equipment's, establishment of connectivity line router, biosensor devices, network operating cabins, remote sensing devices and training facilities that may not be feasible in a wider perspective specially in under developed nation.

- For smooth functioning of eICU continues power supply, dedicated and ultra-fast Internet or LAN connectivity, intact functioning hardware and software's are mandatory, any breech in this algorithm due to machine malfunction or interruption cause by natural calamities might disrupt the entire operating apparatus posing a major risk on the delivery healthcare in a critical ICU specially in times of wide spread pandemic like covid 19 unless a backup apparatus is in place. ${ }^{13}$

- Many of the healthcare professional might not be akin to newer technological advancement and might prefer physical touch and heal strategy in such a scenario the entire essence of eICU is lost.

- For the country's healthcare system to revolutionize, needs to be a balance amongst the number of existing trained doctors to treat patients in eICU setup and for technological advances to offer treatment options.

- If a healthcare professional is situated in a high security zone with jammers and other devices installed that interferes with the data speed and connectivity. Smooth delivery of critical services might be jeopardy.

- High profile data of patients like politicians, celebrities can be hacked for commercial exploitation.

- Though eICU is a revolution in patient care, it cannot exchange the human element in treatment. ${ }^{14}$

\section{DISCUSSION}

The concept of e ICU's although established in some developed and developing countries across the world for managing critical care illnesses by skilled staff from the ease of their home, hasn't gained momentum on a broader scale. Reasons for this could be multi-pronged, be it the challenges related to infrastructure like installation of technologically advanced equipment's containing Biosensing devices, Tele monitor, Router services, Embedded apps or such other gadgets that forms part of eICU functioning, or the deficiency of training or willingness to change from conventional patient management protocols among the skilled staffs and clinicians.

In a pandemic situation like Covid-19, where chances of contracting the illness is pretty high due to highly contagious nature of the virus, where the infection among healthcare workers are increasing on a daily basis due to repeated exposure to Covid-19 patients, where development of complications require ICU management on a large scale in countries like India, Russia, China etc, where the deficiency of PPE and staff is becoming an emerging trend, where treatment costs in private setup are shooting sky-high, most of which is consumed for procuring PPEs and other ICU gadgets for repeated patient interaction to address the emergence and monitor their prognosis in a Covid-19 crisis.

eICU's with their interconnect chain of other ICUs would come to rescue the healthcare system, which can manage a vast majority of ICU admitted patients through their interconnected network of eICUs through the concept of Telemedicine based on Artificial Intelligence and Embedded technology systems. A dedicated, well trained and sophisticated small team of Technically expert intensivist, Physician and few other in-house staff can manage and interact with every individual through their respective monitor and track their vitals, other parameters from the ease of their home. This could prevent undue exposure of Healthcare professionals to the risk of succumbing to Covid-19, make it feasible to handle large group of patients with individualized care and benefit the infrastructure and economy in the long run. Although implementing this technology may face little challenges with procuring advanced gadgets, recruiting and training staff, availability of skilled technicians and initial installation cost, long-term prospects with management of Covid-19 and allied emergencies would prove beneficial. The Union and state governments must take special interest in updating the existing ICUs and converting them into eICU's on a large scale through implementation of nationwide programmes, encouraging public private partnership models to come forward and execute these initiatives. This would treat many patients and prevent intellectual resources from unduly getting infected and losing their lives. 


\section{CONCLUSION}

Telemedicine is rather a debatable concept, but in view of the current crisis situation of Covid-19 pandemic, it appears to be the modality of choice. It's a tool that will enhance the moral delivery of health care. Telemedicine was a bystander of the past, which could be the new hope of future in the segment of essential healthcare delivery with decreased risk of exposure and infection. The stakeholders of medical fraternity must ensure that no harm is caused accidentally or as a result of failure of such facilities. The speedy growth of medical informatics and secondary technologies has expanded the boundaries of critical care medicine. The current healthcare system's current problem is the increasing demand for physician services, and therefore, the growing need for cost suppression will become more complex in future. The e ICU/ tele-ICU model for COVID19 would present a viable and safe means for providing high-quality care to underserved communities. eICU/Tele-ICU's would see substantial growth in width and impact in the future years and will bring a value proposition in the long run. Although, the standard of care, physical touch, consent etc, remains of concern, but the benefits in high risk pandemics is multipronged.Hence the government, regulatory bodies and healthcare management must give a deep thought on revolutionizing age-old ICU models to technologically advanced eICU's/ Tele-ICU's that may cater to a larger population with limited intellectual resources in a crisis like COVID-19 pandemic.

\section{ACKNOWLEDGEMENTS}

We would like to thank Dr. Surendra Naik, MD (Internal Medicine), Dr. HarishH. S, Ophthalmologist Delhi and Dr.M.K. Srinivas, IRMS, Bangalore, for reviewing the article and giving opinions, comments and corrections as appropriate. We extend our thanks to Dr. B. Devanand, Director, VIMS, Ballari, Dr. D. Krishnaswamy, Principal, VIMS, Ballari, Dr. J. Mariraj, Medical Superintendent, VIMS, Ballari, Dr.Y.Vishwanath Professor, Department of Pharmacology, VIMS, Ballari, Dr. Sushil Kumar Varma, Prof \& HOD, Department of Pharmacology, MGIMS, Sevagram, for constantly encouraging us to contribute back to medical fraternity and other Faculties of Department of Pharmacology, VIMS, Ballari for their encouragement.

We acknowledge the immense help received from the scholars whose articles are cited and included in references of this manuscript. We are also grateful to authors / editors / publishers of all those articles, journals and books from where the literature for this article has been reviewed and discussed.

\section{CONFLICT OF INTEREST}

The authors involved in the current study does not declare any competing conflict of interest.

\section{FUNDING AND SPONSORSHIP}

No fund or sponsorship in any form was obtained from any organisation for carrying out this research work.

\section{REFERENCES}

1. Ramanathan K, Antognini D, Combes A, Paden M, Zakhary B, Ogino $\mathrm{M}$ et al. Planning and provision of ECMO services for severe ARDS during the COVID-19 pandemic and other outbreaks of emerging infectious diseases. The Lancet Respiratory Medicine. 2020;8(5):518-526.

2. Singhal T. A Review of Coronavirus Disease-2019 (COVID-19) [Internet]. Indian journal of pediatrics. Springer India; 2020. Available from: https://www.ncbi.nlm.nih.gov/pubmed/32166607. [cited 2020May18]

3. Ing EB, Xu AQ, Salimi A, Torun N. Physician Deaths from Coronavirus Disease (COVID-19). medRxiv. 2020 Jan 1.

4. Yang X, Yu Y, Xu J, Shu H, Liu H, Wu Y, Zhang L, Yu Z, Fang M, Yu T, Wang Y. Clinical course and outcomes of critically ill patients with SARS-CoV-2 pneumonia in Wuhan, China: a single-centered, retrospective, observational study. The Lancet Respiratory Medicine. 2020 Feb 24.

5. Dr Naresh Rathod, Dr Jyothi DB, Dr Fouzia M. Non-Pharmaceutical Interventions For Community Preparedness And Response - Pros And Cons In A Pandemic: IJAR, Volume 10, Issue-4, April 2020;1-3.

6. Serper M, Volk ML. Current and future applications of Telemedicine to optimize the delivery of care in chronic liver disease. Clinical Gastroenterology and Hepatology. 2018 Feb $1 ; 16(2): 157-61$.

7. Scurlock C, Becker C. Telemedicine for Trauma and Emergency: the eICU . Current Trauma Reports. 2016 Sep 1;2(3):132-7.

8. Greenstock L, Woodward-Kron R, Fraser C, Bingham A, Naccarella L, Elliott K, Morris M. Telecommunications as a means to access health information: an exploratory study of migrants in Australia. Journal of public health research. 2012 Dec 28;1(3):216.

9. Luanrattana R. A review of information technology use in medical education: an overview. In2011 7th International Conference on Advanced Information Management and Service (ICIPM) 2011 (pp. 121-124). IEEE.

10. Rashid M., Two decades (1993-2012) of adult intensive care unit design: A comparative study of the physical design features of the best practice examples. Critical care nursing quarterly. 2014 Jan 1;37(1):3-2.

11. COVID C. Global Cases by the Center for Systems Science and Engineering (CSSE) at Johns Hopkins University (JHU). JHU COVID-19 Resource Center. Johns Hopkins Coronavirus Resource Center.

12. Kumar S, Merchant S, Reynolds R. Tele-ICU: efficacy and costeffectiveness approach of remotely managing the critical care. The open medical informatics journal. 2013;7:24.

13. Paul DP, Bailey D, Hairston A, Deslich S, Coustasse A. The Business Case for the Efficiency and Effectiveness of Tele-Intensive Care Units.

14. Lokkerbol J, Adema D, Cuijpers P, Reynolds III CF, Schulz R, Weehuizen R, Smit F. Improving the cost-effectiveness of a healthcare system for depressive disorders by implementing Telemedicine: a health economic modeling study. The American Journal of Geriatric Psychiatry. 2014 Mar 1;22(3):253-62.

15. Nambudiri VE, Sikka N, Choudhri T, Jarrin R, Hale TM, Kvedar JC, Hays H, Carroll M, Ferguson S, Fore C, Horton M. 
Rathod et al.: e-ICU's/Tele ICU's, it's role, advantages over manual icu's and shortcomings in the current perspective of covid-19...

Telemedicine's Challenges to the Medical Profession. Virtual Mentor. 2014 Dec;16:955.

16. Coronavirus in Europe: Thousands of Health Workers Out of Action - The New York Times [Internet]. Available from: https://www.nytimes.com/2020/03/24/world/europe/coronaviruseurope-covid-19.html[cited 2020 Jun 5]

17. Casanova L, Alfano-Sobsey E, Rutala WA, Weber DJ, Sobsey M. Virus transfer from personal protective equipment to health- care employees' skin and clothing. Emerging infectious diseases. 2008 Aug;14(8):1291.

18. Lee SY, Choi SH, Park JE, Hwang S, Kwon KT. Crucial role of temporary airborne infection isolation rooms in an intensive care unit: containing the COVID-19 outbreak in South Korea. Critical Care. 2020 Dec;24(1):1-3. 